\title{
Value of nocturnal oxygen saturation as a screening test for sleep apnoea
}

\author{
B G Cooper, D Veale, C J Griffiths, G J Gibson
}

\begin{abstract}
The sensitivity and specificity of overnight recording of arterial oxygen saturation $\left(\mathrm{SaO}_{2}\right)$ in routine clinical practice was evaluated in 41 subjects who were being investigated for possible sleep apnoea-hypopnoea syndrome. $\mathrm{SaO}_{2}$ was measured with an ear probe oximeter (Biox IIa) and chart recorder during an "acclimatisation" night immediately before a detailed polysomnographic study. The recordings were classified by two observers as positive, negative, or uninterpretable. Twelve of the 41 patients had the obstructive sleep apnoea syndrome when defined in terms of an apnoea-hypopnoea index greater than 15 events an hour on the second night. The sensitivity of nocturnal $\mathrm{SaO}_{2}$ on the acclimatisation night when the diagnostic criterion was an apnoeahypopnoea index of $>5,>15$, and $>25 / \mathrm{h}$ was $60 \%, 75 \%$, and $100 \%$ respectively. Corresponding values for specificity were $95 \%, 86 \%$, and $80 \%$. Oximetry alone therefore allowed recognition of a moderate or severe sleep apnoea syndrome. In routine practice an appreciable number of equivocal results is likely and repeat oximetry or more detailed polysomnography will then be required if clinical suspicion is high.
\end{abstract}

Polysomnographic studies for the detection and classification of sleep apnoea and associated disorders are relatively expensive in cost and time, and are often inconvenient for both subject and observer. In many hospitals measurement of nocturnal oxygen saturation $\left(\mathrm{SaO}_{2}\right)$ by oximetry alone is used as a screening test in patients with suspected sleep apnoea. The aim of this retrospective study was to assess the value of a single overnight oximeter recording in predicting whether sleep apnoea is present.

Respiratory Medicine B G Cooper

$\mathrm{D}$ Veale

G J Gibson

Department of Medical Physics C J Griffiths

Freeman Hospital, Newcastle upon Tyne NE7 7DN

Correspondence to: Mr B G Cooper, Departmen of Medicine, University of Newcastle upon Tyne, Newcastle upon Tyne $\mathrm{NE} 24 \mathrm{HH}$

Accepted 27 April 1991 \begin{abstract}
in ofsible sleep apnoea included in the survey, which looked at the isation" night immediately before a more detailed nocturnal study in relation to the polysomnographic recordings on the second night.

Methods

Forty one subjects (15 female) referred for
\end{abstract}

\section{STUDY DESIGN}

All patients were admitted to hospital for two consecutive nights. Oxygen saturation was recorded with a Biox IIA ear oximeter with the output signal connected to a Rikadenki three channel chart recorder. The recorder speed was set at $12 \mathrm{~cm} /$ hour and the start time was recorded by the subject. The fitting of the ear probe was explained to the subject and this was attached with the assistance of a nurse before the subject went to sleep. Nursing staff checked whether the probe was in place on occasion during the night but the subject was not under constant supervision. Polysomnography on the second night included measurement of chest wall movement (by magnetometers), airflow at the nose and mouth (thermocouples), sleep stage (electroencephalogram (EEG) and electro-oculogram (EOG)), and ear oximetry (Biox IIa). These were recorded on to a multichannel chart recorder and FM tape or on to synchronised video tape. ${ }^{1}$

\section{ANALYSIS}

Oximeter records from the acclimatisation night were coded and analysed "blind" by two experienced observers and classified in one of three categories: positive-sleep breathing disorder present; negative-sleep breathing disorder not present; uninterpretable-technically unsatisfactory or "don't know."

The term sleep breathing disorder was used to imply the presence of either the sleep apnoea or the sleep hypopnoea syndrome. Records regarded as "technically unsatisfactory" included those where the chart recorder had failed or where it was suspected from the pattern of the recording that the oximeter probe had come adrift for a large proportion of the night. A recording was classified as positive on the basis not of exact diagnostic criteria but of "pattern recognition" of repetitive dips in oxygen saturation of more than $5 \%$. The observers were aware of the aim of the study and the two nights' traces were read separately, without knowledge of the recordings on the other night. Disturbance of breathing during sleep was classified from the polysomnographic recordings on the subsequent night by an independent observer on the basis of the frequency of episodes of apnoea and hypopnoea (sleep apnoea-hypopnoea index). Samples of the record were also checked by a second observer. Because different values of the apnoea-hypopnoea index are used in the definition of the syndrome, patients were diagnosed on the basis of an 
Agreement between observers on results of oximetry. Both agreed that the result was positive on 13 occasions. Where one or both reported a negative or uninterpretable record the result was taken as negative ( 28 occasions in all).

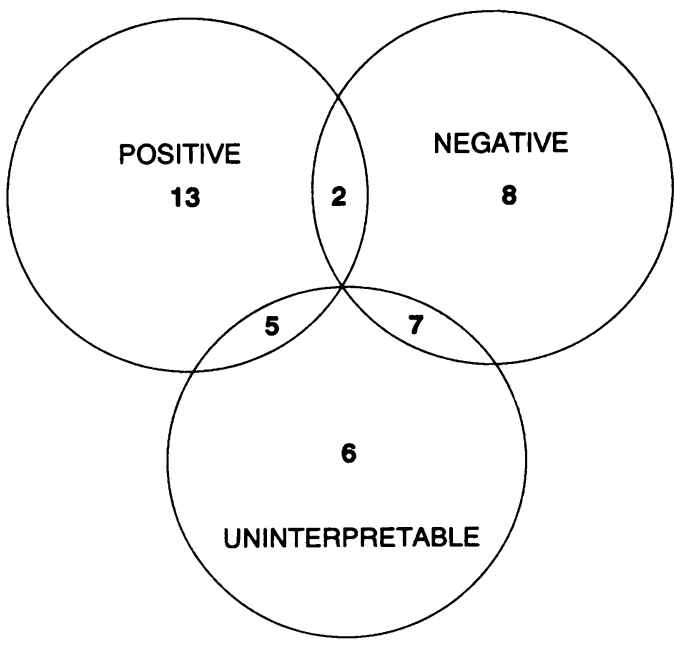

apnoea-hypopnoea index exceeding 5,15 , or 25 events an hour. The information from this definitive sleep study was then used to determine the sensitivity and specificity of the oxygen saturation trace on the first night in detecting the sleep apnoea-hypopnoea syndrome. The sensitivity of oximetry alone for the recognition of the sleep apnoea-hypopnoea syndrome was calculated as the number of true positive $\mathrm{SaO}_{2}$ records divided by the total number of positive definitive (polysomnographic) records-that is, true positive plus false negative $\mathrm{SaO}_{2}$ records. Specificity was defined as the number of the true negative $\mathrm{SaO}_{2}$ records divided by the total number of negative definitive records-that is, true negative plus false positive $\mathrm{SaO}_{2}$ records. In the assessment of the sensitivity and specificity of the overnight oximetry recording on the first night "uninterpretable" traces and those on which the two observers disagreed were regarded as negative. The sensitivity and specificity of $\mathrm{SaO}_{2}$ on the acclimatisation night for detection of sleep apnoea syndrome were determined for apnoea-hypopnoea indices exceeding 5, 15, and 25 events an hour.

\section{Results}

The two observers agreed on $27 / 41(66 \%)$ occasions (figure). Of these records, six were classified by both as uninterpretable, four because of chart recorder or oximeter failure. The observers disagreed on 14 occasions: in 12 of these one or other observer regarded the record as uninterpretable and in two cases there was complete disagreement (figure). The table shows values for the sensitivity and specificity of oximetry as a screening test with the three "diagnostic" thresholds of the apnoeahypopnoea index. For indices exceeding 5, 15, and 25 the sensitivity was $60 \%, 75 \%$, and $100 \%$ respectively, with corresponding specificity of $95 \%, 86 \%$, and $80 \%$ (table). Examination of the results classified as false negatives showed that in each case one or other observer had classified the $\mathrm{SaO}_{2}$ record as "uninterpretable."

\section{Discussion}

A simple screening test for sleep apnoea is desirable because polysomnography is expensive and time consuming. Several screening investigations have been proposed, including laryngeal sounds, ${ }^{2}$ electrocardiography, ${ }^{3}$ flow detection, ${ }^{4}$ and oximetry ${ }^{5}$; but formal evaluation has rarely been performed. The recording of oxygen saturation on the night before polysomnography in this study is likely to be equivalent to the clinical screening method used in many centres.

There is still controversy in published papers ${ }^{6}$ about the defining criterion for the sleep apnoea-hypopnoea syndrome. The originally proposed criterion of more than 5 episodes of apnoea an hour ${ }^{7}$ is now generally regarded as too strict and an apnoea-hypopnoea index above 15 is considered more realistic. ${ }^{8}$ An ideal screening test should have a high sensitivity with a reasonable specificity. Clearly, however, the sensitivity and specificity of screening oximetry are dependent on the criterion used for a positive diagnosis: the lower the value of the apnoea-hypopnoea index chosen for defining the lower limit the higher the specificity of oximetry but the poorer the sensitivity, whereas higher values lead to higher sensitivity but reduced specificity. Our results suggest that oximetry alone allows confident recognition of moderate and severe cases of the sleep apnoeahypopnoea syndrome but it is inadequate for exclusion of milder cases.

Problems in using oximetry for recognising the sleep apnoea syndrome may be technical or physiological. An example of the former is poor contact of the probe with the ear, which occasionally produces signals resembling multiple falls in oxygen saturation; an example of the latter is periodic nocturnal desaturation in patients with chronic airways disease. Such errors increase the false positive results and decrease the specificity. Our criteria were relatively strict in that for a record to be classified as positive both observers had to agree; records where one or both observers considered the saturation record as uninterpretable were taken as negative. Such a policy

Results of polysomnography and classification of oximetry recordings

\begin{tabular}{|c|c|c|c|c|c|c|c|c|}
\hline \multirow{2}{*}{$\begin{array}{l}\text { Criterion } \\
\text { (events per hour) }\end{array}$} & \multicolumn{2}{|c|}{ Polysomnography } & \multicolumn{4}{|c|}{ Oximetry records } & \multirow{2}{*}{$\begin{array}{l}\text { Sensitivity } \\
\text { of oximetry } \\
(T P / T P+F N) \%\end{array}$} & \multirow{2}{*}{$\begin{array}{l}\text { Specificity } \\
\text { of oximetry } \\
(T N / T N+F P) \%\end{array}$} \\
\hline & positive & negative & $T P$ & $F N$ & $T N$ & $F P$ & & \\
\hline \multicolumn{9}{|c|}{ Apnoea-hypopnoea index } \\
\hline $\begin{array}{l}>5 \\
>15 \\
>25\end{array}$ & $\begin{array}{r}20 \\
12 \\
6\end{array}$ & $\begin{array}{l}21 \\
29 \\
35\end{array}$ & $\begin{array}{r}12 \\
9 \\
6\end{array}$ & $\begin{array}{l}8 \\
3 \\
0\end{array}$ & $\begin{array}{l}20 \\
25 \\
28\end{array}$ & $\begin{array}{l}1 \\
4 \\
7\end{array}$ & $\begin{array}{r}60 \\
75 \\
100\end{array}$ & $\begin{array}{l}95 \\
86 \\
80\end{array}$ \\
\hline
\end{tabular}

TP-true positive; FN-false negative; TN-true negative; FP-false positive. 
inevitably reduces the sensitivity of a diagnostic investigation. In the clinical context, however, technical errors or an equivocal result would be regarded as a "negative" outcome and the study would be repeated. One observer produced three more false negative results than the other; in each instance the apnoeahypopnoea index was 5-15/h.

It might be argued that our group of subjects had already been screened by clinical assessment and the referral procedure, but this again reflects the usual clinical circumstances in which oximetry is used as a screening test. Furthermore the oximetry records may be influenced by the "first night" effect of sleep study measurements. ${ }^{9}$ This may mean that, although the diagnosis of the sleep apnoeahypopnoea syndrome is likely to be reliable in severe cases, it will be much less so in patients with milder disease. ${ }^{10}$

In conclusion, nocturnal oxygen saturation alone allowed confident recognition of moderate and severe cases of obstructive sleep apnoea, but it is likely to be inadequate for excluding milder cases in clinical practice. Repeat oximetry or more detailed polysom- nography is then required if clinical suspicion is high.

1 Griffiths CJ, Cooper BG, Gibson GJ. A video system for investigating breathing disorders during sleep. Thorax 1991;46:136-40.

2 Krumpe PE, Cummiskey JM. Use of laryngeal sound recordings to monitor apnoea. Am Rev Respir Dis 1980;122:797-801.

3 Guilleminault C, Connolly S, Winkle R, Melvin K, Tilkian A. Cyclical variation of the heart rate in sleep apnoea syndrome: mechanisms and usefulness of 24 hour electrocardiogram as a screening technique. Lancet 1984;i:126-31.

4 Beckerman RC, Wegmann MJ. A comparison of tracheal breathsounds, airflow and impedance pneumotachography in the detection of childhood apneas. Sleep 1985;8:342-6.

5 Warley ARH, Mitchell JH, Stradling JR. Evaluation of the Ohmeda 3700 pulse oximeter. Thorax 1987;42:892-6.

6 Berry DTR, Webb WB, Block AJ. Sleep apnea syndrome. A critical review of the apnea index as a diagnostic criterion. Chest 1984;86:529-31.

7 Guilleminault C, van den Hoed J, Mitler MM. Clinical overview of the sleep apnea syndromes. In: Guilleminault C, Dement WC. eds. Sleep apnea syndromes. New York: Alan R Liss, $1978 ; 1-12$.

8 Gould GA, Whyte KF, Rhind GB, et al. The sleep hypopnea syndrome. Am Rev Respir Dis. 1988;137:895-8.

9 Agnew HW, Webb WB, Williams RL. The first night effect: an EEG study of sleep. Psychophysiology. 1966;2:263-6.

10 Wittig RM, Romaker A, Zarick FJ, Roehrs TA, Conway WA, Roth T. Night to night consistency of apneas during sleep. Am Rev Respir Dis 1984;129:244-6. 